Research paper

\title{
Serum-based microRNA signature predicts relapse and therapeutic outcome of adjuvant chemotherapy in colorectal cancer patients
}

\author{
Dengbo Ji ${ }^{\mathrm{a}, 1}$, Meng Qiao ${ }^{\mathrm{a}, 1}$, Yunfeng Yao ${ }^{\mathrm{a}, 1}$, Ming $\mathrm{Li}^{\mathrm{a}}$, Hailong Chen ${ }^{\mathrm{b}}$, Qi Dong ${ }^{\mathrm{b}}$, Jinying Jia ${ }^{\mathrm{a}}$, Xinxin Cui ${ }^{\mathrm{a}}$, \\ Zhaowei $\mathrm{Li}^{\mathrm{a}}$, Jinhong Xia ${ }^{\mathrm{a}}$, Jin $\mathrm{Gu}^{\mathrm{a}, \mathrm{a}, \mathrm{d}, *}$
}

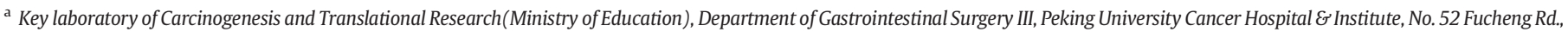
Haidian District, Beijing 100142, China

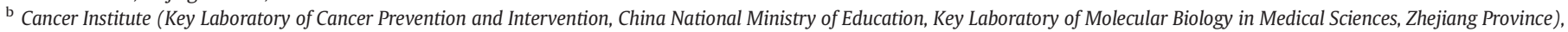
The Second Affiliated Hospital, Zhejiang University School of Medicine, Hangzhou, Zhejiang 310009, China

c Peking-Tsinghua Center for Life Sciences, China

d Peking University S.G. Hospital, China

\section{A R T I C L E I N F O}

\section{Article history:}

Received 5 July 2018

Received in revised form 8 August 2018

Accepted 14 August 2018

Available online 27 August 2018

\section{Keywords:}

Serum-based microRNA signature

Colorectal cancer

Adjuvant chemotherapy

Prognostic biomarker

\begin{abstract}
A B S T R A C T
Background: Approximately $60 \%$ of patients with colorectal cancer (CRC) undergo either local recurrence or distant metastases after surgery. Current prognostic biomarkers are insufficient to predict recurrence of CRC and provide little forecast information about what patients are likely to receive benefit from the adjuvant chemotherapy. As microRNAs (miRNAs) constantly exist in human serum and being used to predict the prognosis of a various cancers, this study was designed to identify miRNA-based circulating biomarkers to predict clinical outcomes of CRC.

Methods: A serum-focused miRNA expression was used to investigate if miRNA expression profiles could predict the clinical outcomes of patients with CRC. We created miRNA signature profiles associated in the training set $(n=40)$, and further validated its prediction in two independent testing cohorts.

Results: Using Cox regression and risk-score analysis, we identified a four-miRNA signature (miR-652-3p, miR342-3p, miR-501-3p and miR-328-3p) for the prediction of tumor relapse and the overall survival(OS) of patients with CRC in the training set $(n=40)$. This miRNA signature was further validated in a testing set $(n=226)$ and another independent cohort $(n=56)$. A high-risk signature score was significantly associated with CRC tumor recurrence and poor treatment outcome. Multivariable Cox regression models indicated that the risk score, based on the four-miRNA signature, was an independent prognostic classifier for patients with CRC.

Conclusions: The serum miRNA signature may serve as a minimally invasive predictor for tumor relapse and treatment outcome in patients with CRC and provide a useful reference for treatment selection.
\end{abstract}

(C) 2018 The Authors. Published by Elsevier B.V. This is an open access article under the CC BY-NC-ND license (http://creativecommons.org/licenses/by-nc-nd/4.0/).

\section{Introduction}

Colorectal cancer (CRC) is one of the most common gastrointestinal malignancies worldwide. CRC is an important cause of cancer mortality and remains a public health burden. As the main treatment method surgical resection is performed, often combined with chemotherapy and/or radiotherapy, for CRC patients. Approximately $60 \%$ of patients undergo local recurrence or distant metastases after surgery [1]. Although the American Joint Committee on Cancer (AJCC) TNM classification is a

\footnotetext{
* Corresponding author at: Key laboratory of Carcinogenesis and Translational Research(Ministry of Education), Department of Gastrointestinal Surgery III, Peking University Cancer Hospital \& Institute, No. 52 Fucheng Rd., Haidian District, Beijing 100142, China.

E-mail address: zlguj@bjmu.edu.cn (J. Gu).

${ }^{1}$ These authors contributed equally to this study.
}

valuable tool for CRC staging and a specific treatment selection, patients at similar clinical stage can have very different outcomes. Therefore, it is highly desirable to identify biomarkers that can aid in the identification of high-risk patients.

Carcinoembryonic antigen (CEA) is the most frequently used marker to predict CRC prognosis [2]. However, CEA provides limited predictive information of tumor recurrence in primary CRC and is not able to foresee which patients are likely to benefit from chemotherapy. Thus, prognostic molecular biomarkers would be able to guide treatment selection and adjust the therapeutic regimes based on fluctuation of the test results from each patient. MicroRNAs (miRNAs) are important regulators of oncogene and tumor suppressor gene expression during tumor formation and progression [3]. MiRNAs are stable and easily detectable in the serum or plasma of patients with cancer, and are reportedly associated with clinicopathologic variables and prognosis [4-6]. Several 


\section{Research in context}

\section{Evidence and motivation of this study}

Approximately $60 \%$ of patients with colorectal cancer (CRC) undergo either local recurrence or distant metastases after surgery. Current prognostic biomarkers are insufficient to predict recurrence of CRC and provide little forecast information about what patients are likely to receive benefit from the adjuvant chemotherapy. After a thorough literature search and review, we found that several studies have highlighted the diagnostic and prognostic utility of circulating miRNA levels in CRC. These existing literatures often enrolled both non-metastatic and metastatic CRC patients. No previous serum miRNAs were focused on predicting the prognosis and therapeutic outcome in stage I-III CRC patients.

\section{Added value of this study}

The major valuable discoveries from this study are summarized below:

1. A serum-based four-miRNA signature which could predict the relapse and the overall survival of stage I-III CRC was identified and validated. The patients with high risk scores had a poor prognosis.

2. The four-miRNA signature correlated with stage I-III CRC therapeutic outcome following adjuvant chemotherapy. The patients with high risk scores did not benefit from current adjuvant chemotherapy.

Implications of all the available evidence

Accurately predicting the prognosis at the time of diagnosis is critical for clinicians to tailor the treatment plan for maximal efficacy and to determine surveillance strategies. Our serum-based fourmiRNA signature may provide a better identification of the patients who could most likely be responding to the adjuvant chemotherapy.

miRNAs were dysregulated in the plasma of patients with CRC. miR-29a and -92a were reported to be significantly elevated in advanced adenomas and CRC plasma when compared with normal controls [7,8]. Circulating miRNAs could be potential non-invasive molecular markers for CRC screening.

In this study, we investigated the prognostic role of human miRNAs in serum samples derived from patients with primary CRC and developed a miRNA signature which was associated with early recurrence and poor outcome in patients with primary CRC.

\section{Materials and methods}

\subsection{Patients}

A total of 322 patients with stage I-III CRC whom preoperative serum could be collected were included in this retrospective study. Patients with stage I-III colorectal cancer, and with clinicopathological characteristics and follow-up information available, were included. We excluded patients if they had any other malignant disorders or other chronic diseases, previous treatment with any anticancer therapy, presence of any tumor type other than adenocarcinoma or mucinous carcinoma, and familial adenomatous polyposis CRC. We also excluded patients if they had no blood sample from initial diagnosis or the blood samples were hemolytic. All patients underwent a radical resection surgery. Adjuvant chemotherapies were recommended for patients with stage III and high-risk stage II disease after surgical resection. The chemotherapy regimens were primarily fluorouracil-based, with or without leucovorin, levamisole, or oxaliplatin. All patients were reevaluated at 3 month intervals for 1 year and every 6 months thereafter. The evaluations included medical history, physical examination, measurements of the serum carcinoembryonic antigen and carbohydrate antigen 199 levels at every follow-up visit. Colonoscopies were performed once a year. Follow-up computed tomography of chest, abdominal and pelvis was sceheduled every 6 months for first 3 years and annually thereafter.

Patients who developed distant metastases within 36 months after removal of the primary tumor were considered positive for tumor relapse, whereas patients without distant metastases during the same time frame were defined as non-relapsing. Relapse was confirmed by computed tomography (CT) imaging or other imaging techniques.

This research was approved by the ethics committee of the Health Science Center of Peking University and the Oncology Center at Peking University and Zhejiang University and in accordance with the Declaration of Helsinki. Written informed consent was obtained from all participants before enrollment to the study.

Circulating biomarkers were tested using serum samples from patients with and without relapsing ( 20 samples for each group). To validate those biomarkers, a predictive risk score was developed and tested in a validation cohort ( $n=226$, from the Peking University Cancer Hospital \& Institute) and an additional independent cohort ( $n=56$, from the Second Affiliated Hospital of Zhejiang University School of Medicine). All clinicopathologic characteristics are reported in Supplementary Table 1 . We also examined the prognostic impact of the miRNAs based on overall survival with a minimum of 60 months follow-up.

\subsection{Sample collection and RNA isolation}

Serum samples were collected from 322 patients with stage I-III CRC prior to any treatments. After informed consent was obtained, $3.5 \mathrm{~mL}$ of venous blood was collected prior to surgery from each patient and processed within $1 \mathrm{~h}$ based on protocols of NCl's Early Detection Research Networks (EDRN) [9]. All samples were checked for hemolysis using the Harboe's spectrophotometric methods [10] and hemolytic samples with free hemoglobin concentration $>0.6 \mathrm{~g} / \mathrm{L}$ [11]were excluded. Serum was span at $3000 \mathrm{~g}$ for $5 \mathrm{~min}$ at $4{ }^{\circ} \mathrm{C}$ to remove debris. Samples were aliquoted and stored in RNase-free tubes at $-80^{\circ} \mathrm{C}$ until use. Total RNA was extracted from $200 \mu \mathrm{L}$ of serum using the miRCURYTM RNA Isolation Kit - Biofluids (Exiqon, Copenhagen, Hovedstaden, Denmark), following the manufacturer's guidelines and Exiqon's specific application instructions. Briefly, a QIAzol mixture containing MS2 bacteriophage RNA (Roche, Mannheim, Baden-Württemberg, Germany) was added to serum samples before RNA purification. Total RNA was eluted in RNase-free water and stored at $-80^{\circ} \mathrm{C}$ until use.

\subsection{Quantitative real-time polymerase chain reaction ( $q R T-P C R)$ analysis and miRNA expression}

Expression of miRNAs from the training cohort serum samples was evaluated using miRCURYTM LNA Universal RT miRNA PCR (Exiqon, Denmark). All the miRNAs were polyadenylated and reverse transcribed into cDNA in a single reaction step. Before committing sample RNA to profiling on microRNA qPCR panels, we checked the quality of total RNA isolated from the cell-free serum using three parameters: 1. Presence of typical serum and plasma microRNAs. Good microRNAs for this pre-study include the following microRNAs which are typically detected at medium to high levels in serum and plasma samples: hsamiR-103-3p, hsa-miR-191-5p, miR-423-3p. 2. Absence of inhibitors of the cDNA synthesis and the PCR enzyme. We included the RNA spikein in the RT and analyzed this target in parallel to the microRNA assays. 3. Absence of RNA from cells not removed before extraction. We 
evaluated this by assaying the microRNAs expressions which are highly expressed in red and white blood cells, such as hsa-miR-16-5p and hsamiR-451a. Samples with low RNA yield can be excluded from further studies based on low signal in this pre-study, and samples containing PCR inhibitors will show dilution curves without the expected linear relationship between sample input and signal.

Each miRNA was assayed by qRT-PCR on miRNA Ready-to-Use PCR which contained human serum/plasma miRNA PCR Panel I and Panel II V1, including 175 miRNAs (Exiqon). The samples with hemolysis were excluded by analyzing miR-451a and miR-16-5p expression levels. Negative controls without template were performed. Amplification was performed in an ABI 7500 Fast PCR machine. The real-time PCR cycle conditions were as follows: polymerase activation/denaturation at 95 ${ }^{\circ} \mathrm{C}$ for $10 \mathrm{~min}$; 40 amplification cycles at $95^{\circ} \mathrm{C}$ for $10 \mathrm{~s}, 60{ }^{\circ} \mathrm{C}$ for $1 \mathrm{~min}$. The melting analysis was added finally to evaluate the specificity of PCR products. An RNA spike-in (UniSp6) and a DNA spike-in (Sp3) were applied in the panel as quality controls of the cDNA synthesis reaction and the PCR. The miRNAs with cycle threshold values above 37 were excluded from additional statistical analysis, according to the MIQE guidelines [12]. Expression of the miRNAs in all samples $(n=$ 40) were analyzed using ExiqonGenEx software (Exiqon, Denmark). The best reference gene(s) were selected based on a determination of the most stably expressed gene(s) using GeNorm and NormFinder both tools that are integrated within GenEx data analysis software. GeNorm and NormFinder both look at gene expression variance to choose the most stably expressed genes. M-value in geNorm and standard deviation (SD) in NormFinder indicate the variability of each gene. In this study, the expression levels of miR-103-3p and miR-93$5 \mathrm{p}$ were stable and used as the reference genes for normalization(Supplementary Fig. 1).

In the training data set, 4 of 175 miRNAs were significantly associated with patient survival, as identified by Cox proportional hazards regression. Validation was performed using Pick-\&-Mix PCR (Exiqon, Denmark). Briefly, the cDNA from the RT reaction was diluted 100 times and then combined with $S Y B R \circledast$ Green Master Mix. The mixture was added to a PCR plate containing Locked Nucleic Acid (LNA) primer sets. Real-time PCR was performed on an ABI 7500 Fast PCR machine. Details of experiment methods are described in Supplementary data. The primers of the four miRNAs are listed in Supplementary Table 2.

\subsection{Ingenuity ${ }^{\circledR}$ Pathway Analysis (IPA)}

To investigate the biological interactions of the four miRNAs, we used in silico analysis to find predictive targets and identify their corresponding networks. The predicted targets and networks were generated through the use of QIAGEN's IPA. The predicted networks describe functional relationships between miRNAs and genes based on a knowledgebase of predicted and experimentally observed relationships [13]. Using IPA's miRNA target filter, we generated a list of predicted mRNA targets for each miRNA. The list was then reduced to the mRNAs having verified targets in the IPA database. Then a canonical pathway analysis was performed for the listed mRNA targets. Details about IPA are described in Supplementary data.

\subsection{Statistical analysis}

Early screening study was to examine the expression level of each miRNA. Hazard ratios from the univariable Cox regression analysis were used to identify miRNAs associated with death due to cancer recurrence or any cause. A permutation test was performed to further validate and select miRNAs [14]. The level of statistical significance was set at $p<0.05$.

In the training data set, the Cox proportional hazards regression analyses revealed that several miRNAs were significantly associated with patient survival. These candidate miRNAs were used to construct several signatures to simulate the strength and association of each miRNA with the overall survival. A risk score, weighted by the regression coefficients estimated from the aforementioned Cox regression analyses, was calculated for each patient [15]. The several miRNAsbased signatures were further validated in testing cohort and an independent cohort. Only the miRNA signature constructed by a four miRNAs was verified as a good predictor of tumor relapse and treatment outcome for patients. From the four miRNA signatures, the risk score for each patient was generated from the following justification: $(-0.388 \times$ expression level of hsa-miR-652-3p) $+(-0.875 \times$ expression level of hsa-miR-342-3p) $+(-0.171 \times$ expression level of hsa-miR-501-3p) $+(-0.298 \times$ expression level of hsa-miR-328-3p). Patients with higher risk scores were expected to have a poor survival outcome.

Using the median miRNA signature risk score as the cut-off point, we divided patients in the training data set into two groups: a highrisk group and a low-risk group. The overall survival and relapse-free survival curves were plotted using Kaplan-Meier analysis, and the log-rank test was used to detect the significant difference between the high- and low-risk patients. In the validation stage, the effectiveness of our miRNA signature risk-score model was further examined in a testing set $(n=226)$ and an additional independent set of samples $(n=56)$. Cox univariable and multivariable proportional hazards models were used to estimate the hazard ratio for each marker, which investigated if the miRNA signature was an independent predictor of overall survival and disease-free survival (DFS) in patients with CRC. DFS was defined as the time from the date of surgery until first recurrence or death due to any cause, whichever was observed first. OS was defined as the time from the date of surgery until death from any cause. The Cox regression model requires two assumptions: 1. Non-informative censoring. Censoring should be independent of failure time; in other words, participants who drop out of the study or are lost to follow-up should do so due to reasons unrelated to the study. 2. Constant relative hazard. Each variable has been tested using the global test for proportionality to get a chi-sq result and a $p$-value. The p-value should be $>0.05$. To evaluate the prognostic potential of the miRNA signature risk-score model, receiver operating characteristic curve (ROC) and area under the curve (AUC) were calculated. For evaluation of the effect of adjuvant chemotherapy, DFS was also used as a determinant of response. DFS was defined as the time from the start of chemotherapy to the time of disease relapse or of death from any cause, whichever was observed first [16]. Comparisons in miRNAs expression level between healthy control and CRC patients were performed using the nonparametric Mann-Whitney $U$ test. Differences in clinicopathological characteristics between high risk score and low risk score patients were assessed using Chi-square test. All tests were two-sided and the level of statistical significance was set at $p<0.05$. Statistical analyses were performed using GraphPad Prism ${ }^{\circledR}$ version 5.

\section{Results}

\subsection{Detection of the serum four-miRNA signature in the training data set}

In the training data set $(n=40)$, the Cox proportional hazards regression revealed that four miRNAs (hsa-miR-328-3p, hsa-miR-652$3 p$, has-miR-342-3p and hsa-miR-501-3p) were significantly associated with patient survival (Supplementary Table 3). These four miRNAs were employed to construct a risk-score formula for survival prediction. The expression values of these 4 miRNAs in training set were shown in Supplementary Table 4.

\subsection{Association between the serum four-miRNA signature and patient} prognosis in the training set

Using the four-miRNA signature risk-score formula, the risk scores for all patients in the training set were calculated. Using the median 
risk score as a cutoff point, the patients were divided into a high-risk group and a low-risk group. Supplementary Table 1 shows the clinical characteristics of the 40 patients in the training set. Patients with a high risk score had shorter median OS than the patients with a low risk score ( $p=0.035$, the log-rank test) (Fig. 1a). Patients with a high-risk score tend to have a shorter median relapse-free survival than patients with a low risk score $(p<0.0001$, the log-rank test) (Fig. 1b). The expressions of the four miRNAs in healthy controls ( $n=$ 30) were also examined. No significant difference in the expression levels of these miRNAs was found between healthy controls and CRC patients (Supplementary Fig. 2).
3.3. Validation of the serum four-miRNA signature for relapse and survival prediction in the testing set

We further calculated the risk score for the 226 patients in the testing set and divided them into the high- or low-risk group based on the same cutoff point used in the training set. Similar to the training set, a high-risk miRNA signature was negatively associated with OS ( $p=$ 0.007, the log-rank test) and DFS ( $p<0.0001$, the log-rank test) (Fig. 1c, d).

Fig. 2 further shows the distribution of risk scores, the survival status and tumor miRNA expression of the testing set with 226 patients. More

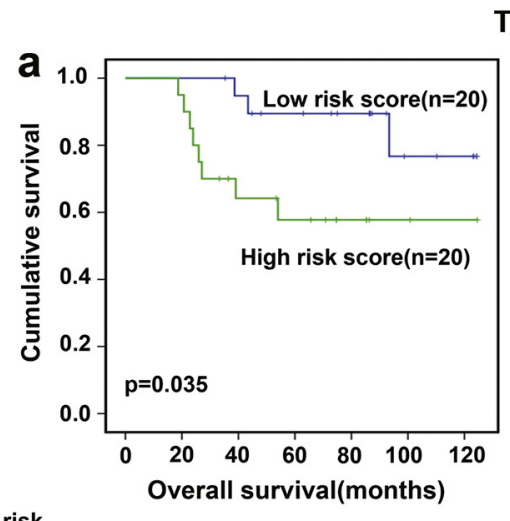

Training set

No. at risk
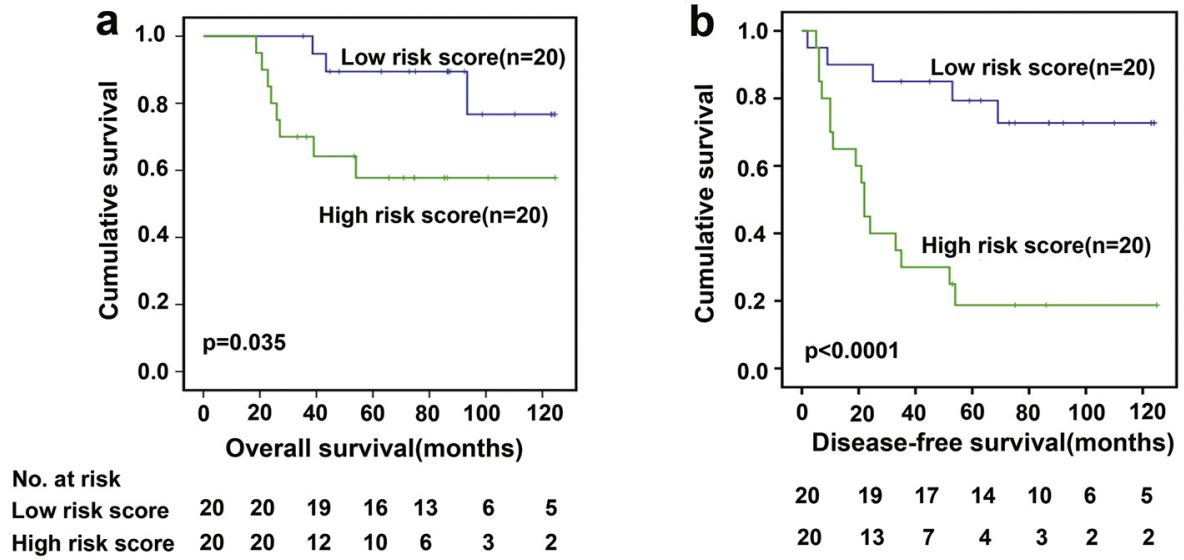

High risk scor

202

210

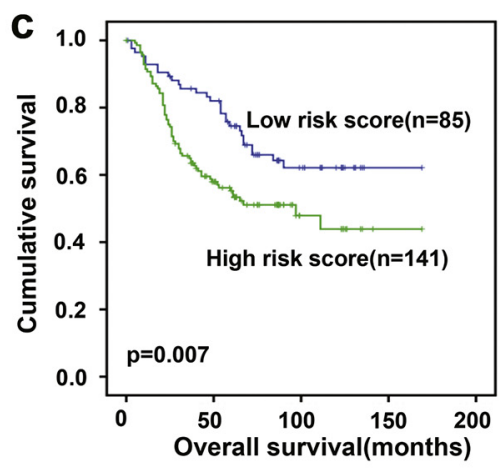

Testing set

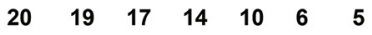

$\begin{array}{lllllll}20 & 13 & 7 & 4 & 3 & 2 & 2\end{array}$

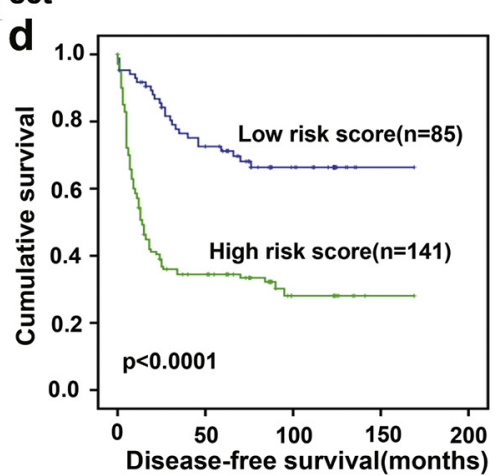

No. at risk

$\begin{array}{llllll}\text { Low risk score } & 85 & 68 & 29 & 2 & 1\end{array}$

$\begin{array}{lllllll}\text { High risk score } & 140 & 70 & 14 & 2 & 1\end{array}$

$\begin{array}{lllll}84 & 56 & 26 & 2 & 1\end{array}$

$\begin{array}{lllll}136 & 44 & 12 & 2 & 1\end{array}$

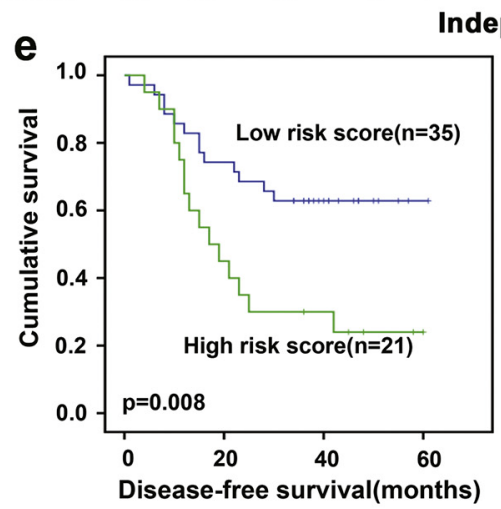

No. at risk

$\begin{array}{lrrrr}\text { Low risk score } & 35 & 27 & 13 & 2 \\ \text { High risk score } & 21 & 11 & 6 & 1\end{array}$

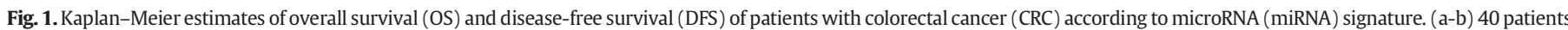
in the training data set; (c-d) 226 patients in the testing data set; (e) 56 patients in the independent cohort. 


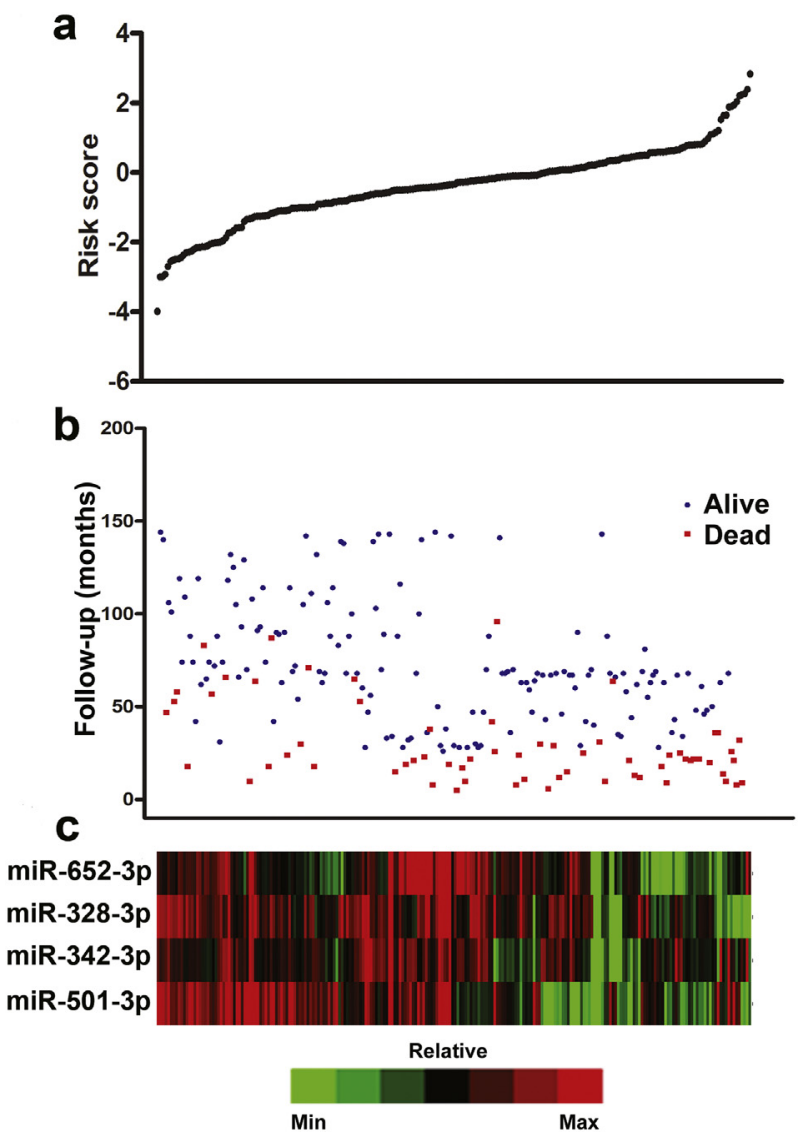

Fig. 2. Risk-score analysis of 226 patients with CRC. (a) MiRNA risk-score distribution; (b) patient survival status; (c) color-gram of miRNA expression profiles of patients with CRC. Rows represent miRNA; columns represent patients.

deaths occurred in patients with high risk score group than those with low risk scores.

In order to investigate if the identified miRNA signature could distinguish the high- and low-risk groups in each stage (CRC stage II or III) and predict the patient's prognosis, we performed a log-rank test for each stage. In each stage, the miRNA signature was significantly associated with DFS in those patients ( $p=0.044$ for stage II; $p<0.0001$ for stage III, the log-rank test) (Fig. 3).

a

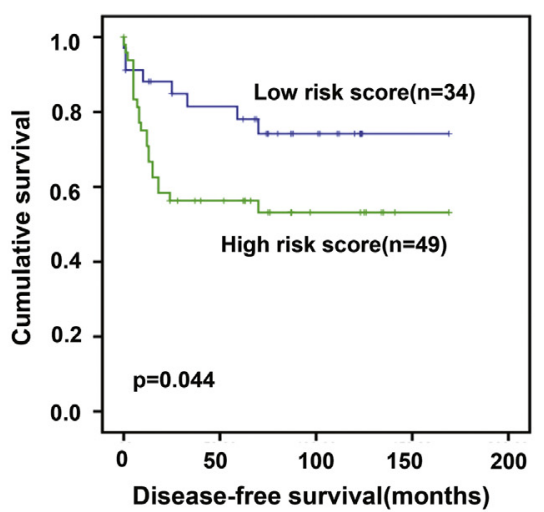

To determine whether there was prognostic association among the expression of CEA, CD44, or p53 status with DFS, we plotted Kaplan-Meier survival curves; the curves indicated a significant difference among DFS and the expression of post-operative serum CEA ( $p=0.028$, the log-rank test) and CD44 expression in CRC tissues $(p=0.040$, the log-rank test). There was no significant correlation between DFS and p53 status (Supplementary Fig. 3).

Multivariable analysis was performed to determine the prognostic value of miRNA signature using the Cox proportional hazard model. The risk variables examined included miRNA signature score, as well as factors known to significantly affect the outcome of CRC such as age and gender of patients, histological type, differentiation, venous invasion, tumor location, surgical-pathological staging, chemotherapy, preop CEA, post-op CEA, CD44 and p53 status. For DFS, in the univariable analysis, MiRNA expression signature [Hazard ratio (HR) 4.552; 95\% confidence interval (CI) 2.702 to $7.671 ; p<0.0001$, Cox univariable proportional hazards models], TNM staging (HR 2.364; $95 \%$ CI 1.553 to 3.599; $p<0.0001$, Cox univariable proportional hazards models), and post-op CEA (HR 1.634; 95\% CI 1.018 to $2.622 ; p=0.042$, Cox univariable proportional hazards models) were significantly associated with DFS. In the final Multivariable Cox regression model, the serum miRNA signature $(\mathrm{HR}=2.675 ; 95 \% \mathrm{CI}=1.41-5.076 ; p=0.003$, Cox multivariable proportional hazards models) and TNM stage (HR $=$ $2.063 ; 95 \% \mathrm{CI}=1.122-3.795 ; p=0.02$, Cox multivariable proportional hazards models) were both prognostic factors associated with DFS independent of other clinical covariates (Supplementary Table 5).

For OS, in the univariable analysis, MiRNA expression signature (HR 2.56; $95 \% \mathrm{CI} 1.319$ to $4.9661 ; p=0.005$, Cox univariable proportional hazards models), TNM staging HR 2.233; $95 \%$ CI 1.225 to $4.069 ; p=$ 0.009 , Cox univariable proportional hazards models), Differentiation (HR 2.039; 95\% CI 1.131 to 3.677; $p=0.018$, Cox univariable proportional hazards models) and Venous invasion (HR 1.785; 95\% CI 1.002 to $3.182 ; p=0.049$, Cox univariable proportional hazards models) were significantly associated with OS. In the final Multivariable Cox regression model, MiRNA expression signature was associated with a poor survival prognosis (HR 2.524; 95\% CI 1.287 to 4.949; $p=0.007$, Cox multivariable proportional hazards models) independent of other clinical covariates (Supplementary Table 6). Similar results were also found in the training set (Supplementary Tables 7 and 8).

The genuineness of the predictive value from the serum four-miRNA signature was accessed with recurrent and non-recurrent CRC patients. ROC analyses showed that the serum four-miRNA signature was a useful predictive biomarker for tumor relapse, with ROC curve areas of 0.7427 ( $95 \% \mathrm{CI}=0.6777-0.8077$ ). Given the cut-off value of -0.6672 for the

b TNM stage 3

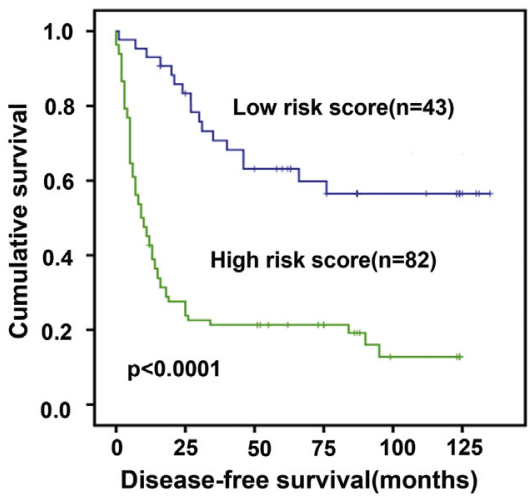

No. at risk

$\begin{array}{lllcll}\text { Low risk score } & 33 & 25 & 13 & 3 & 1 \\ \text { High risk score } & 47 & 24 & 8 & 2 & 1\end{array}$

$\begin{array}{llllll}43 & 34 & 25 & 19 & 16 & 4 \\ 79 & 22 & 18 & 12 & 4 & 2\end{array}$




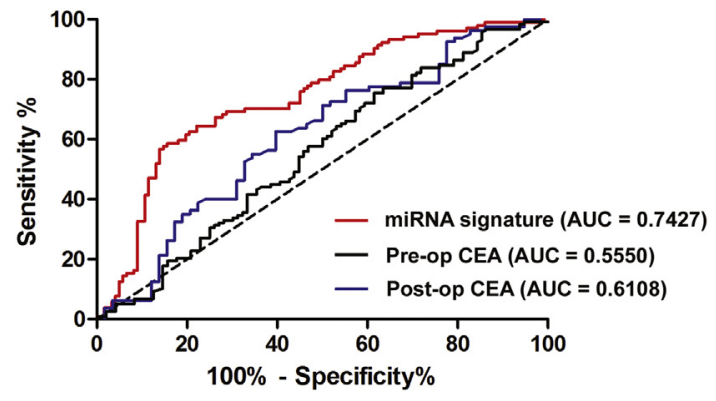

Fig. 4. Receiver operating characteristic (ROC) curve for primary CRC recurrence by the miRNA signature and carcinoembryonic antigen (CEA). ROC curves generated using the prognosis information and expression levels of the miRNA signature, pre-, and postoperative CEA are able to discriminate between patients with recurrence and those without recurrence in the testing cohort. The miRNA signature has the strongest predictive value (area under the curve $[\mathrm{AUC}]=0.7427$ ) to discriminate those patients.

serum four-miRNA signature, the sensitivity was $58.65 \%$ (95\% CI = $48.58 \%-68.23 \%)$ and the specificity was $84.63 \%$ (95\% CI $=76.75 \%-$ 90.36\%)., respectively. The ROC curve areas for pre-operative CEA and post-operative CEA were $0.555(95 \% \mathrm{CI}=0.4765-0.6336)$ and 0.6108 $(95 \% \mathrm{CI}=0.5139-0.7077)$, respectively. Given a cut-off value of 2.205 $\mathrm{ng} / \mathrm{mL}$ for pre-operative CEA and $2.62 \mathrm{ng} / \mathrm{mL}$ for post-operative CEA, the sensitivity and specificity were $75.42 \%(95 \% \mathrm{CI}=66.65 \%-82.88 \%)$ and $38.54 \%$ (95\% CI $=28.78 \%-49.03 \%$ ), 62.5\% (95\% CI $=50.96 \%-$ $73.08 \%$ ) and 60.34\% (95\% CI = 46.64\%-72.95\%), respectively (Fig. 4).

3.4. Revalidation of the serum four-miRNA signature for relapse prediction in an independent cohort

To reconfirm our miRNA signature in an independent cohort, we included 56 patients with CRC from the Second Affiliated Hospital of Zhejiang University School of Medicine. Supplementary Table 1 shows the clinical characteristics of the patients in the independent cohort. Patients were divided into a high- or low-risk group based on their miRNA signature risk scores. The patients with a high-risk miRNA signature tend to have a shorter relapse-free survival than those with low-risk miRNA signatures ( $p=0.008$, the log-rank test) (Fig. 1e).

The relationships between the serum miRNAs signature and clinicopathological characteristics of CRC patients in the training set, the testing set, and the independent set were also analyzed. There was significant correlation between the serum miRNAs signature and distant metastasis in three cohorts $(p=0.0004, p=0.0001$ and $p=$ 0.026 , respectively. Chi-square test). No significant association was observed between the serum miRNAs signature and any other clinicopathological factors in testing cohort and independent cohort. In the training set, the serum miRNAs signature was associated with TNM stage ( $p=$ 0.049 , Chi-square test) and T stage ( $p=0.019$, Chi-square test) (Supplementary Table 9 ).

\subsection{Correlation between the serum four-miRNA signature and therapeutic} outcome following adjuvant chemotherapy

The association between the serum four-miRNA signature and therapeutic outcome was analyzed in patients treated with adjuvant chemotherapy in the testing cohort. The chemotherapy regimens were primarily fluorouracil-based, with or without leucovorin, levamisole, or oxaliplatin. For individuals who received adjuvant therapy, a high risk score was associated with poor therapeutic outcome $(p<0.0001$, Kaplan-Meier log-rank test) (Fig. 5a). In stage II or III, the miRNA signature was also negatively associated with the DFS in CRC patients ( $p=$ 0.028 for stage II; $p<0.0001$ for stage III, the log-rank test) (Fig. 5b, c). Similarly, a high risk score was associated with a poor OS prognosis in stage II or III ( $p=0.008, p=0.029$, the log-rank test, respectively) (Fig. $5 d$, e). Therefore, the serum four-miRNA signature emerged as a validated independent predictor of the response to adjuvant chemotherapy.

\subsection{Functional analysis of the four-miRNA signature}

To examine possible biological functions associated with the serum four-miRNA signature, IPA core analysis was employed to identify the target genes of the four highly predicted and experimentally observed miRNAs. The IPA results suggested that the members of the fourmiRNA signature were involved in a number of biological functions relevant to malignant solid tumors, breast or colorectal cancer, survival of myeloid progenitor cells, neoplasia of cells, colorectal neoplasia, squamous cell carcinoma, cancer of cells, colorectal cancer, and abdominal cancer. A majority of these highly predicted or experimentally observed genes were involved in malignant solid tumors and colorectal cancer (Supplementary Fig.4a). In addition, canonical pathway analysis of these four miRNAs indicated the involvement of cancer drug resistance via drug efflux, regulation of actin-based motility by Rho, actin cytoskeleton signaling, integrin signaling, and High Mobility Group Protein B1 (HMGB1) signaling pathways (Supplementary Fig. 4b-f). Our in silico results suggested that the four-miRNA signature was associated with biological functions correlated with tumor cell mobility and drug resistance.

To clarify these mRNA targets expressions in colorectal cancer and their connection with the four miRNAs, we further used TCGA miRNAseq and mRNA-seq data set to analyze the correlation between miRNAs expression and these targets. Among these predictive targets, some mRNAs showed negatively/positively correlated with its corresponding miRNA. All these analysis results are shown as Supplementary Fig. 5.

\section{Discussion}

In this study, we demonstrated that circulating miRNAs measured at the time of diagnosis could be used to predict disease progression in primary CRC. The expression pattern of four miRNAs (miR-342-3p, miR652-3p, miR-501-3p, and miR-328-3p) was consistently altered in patients with a poor outcome. The serum four-miRNA signature was associated with DFS and OS of stage I-III CRC. It was also correlated with the therapeutic outcome of adjuvant chemotherapy. Our data showed that the serum-based four-miRNA signature was informative in predicting the clinical outcome of patients with CRC: The patients with a highrisk score had a correspondingly high likelihood of aggressive disease and resistance to adjuvant chemotherapy.

Although current guidelines indicate that adjuvant chemotherapy should be considered for patients with stages II and III CRC with poor prognostic features, it is estimated that $>80 \%$ of patients with CRC may not receive a great benefit from the adjuvant treatment [17]. Accurately predicting the prognosis at the time of diagnosis is critical for clinicians to tailor the treatment plan for maximal efficacy and to determine surveillance strategies. Our four-miRNA signature may provide a better identification of the patients who could most likely be responding to the adjuvant chemotherapy.

Prognostic markers in CRC have been sparseness and the available ones are not easily translated into the clinical setting. In addition, molecular heterogeneity induced by tumor biopsies could affect the interpretation of the results [18]. The miRNA markers present in the peripheral circulation would be more directly and easily applicable to the clinical setting.

Several studies have highlighted the diagnostic and prognostic utility of circulating miRNA levels in CRC. Recent findings suggest that the levels of miR-1290, miR-92a, miR-122, miR-200, miR-21, miR-203 and a serum-based signature might have prognostic significance in CRC patients [19-25]. The variability exist among studies due to multiple factors, including the number of samples, the constitute of the training cohort and testing cohort, the number of miRNA tested, the technique used for testing and the reference genes used. 
a Chemotherapy

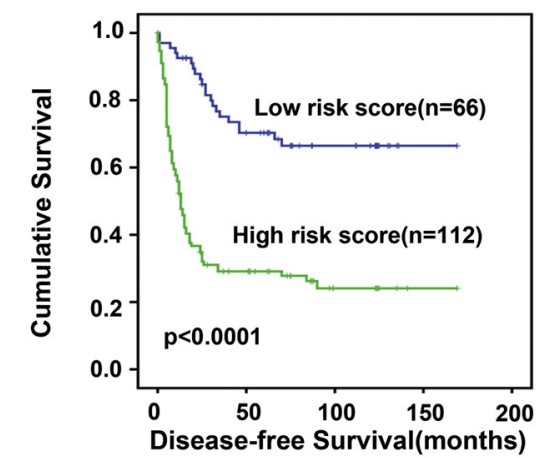

No. at risk

$\begin{array}{cccccc}\text { Low risk score } & 66 & 43 & 29 & 2 & 1 \\ \text { High risk score } & 108 & 29 & 10 & 2 & 1\end{array}$

b TNM stage 2 with chemotherapy

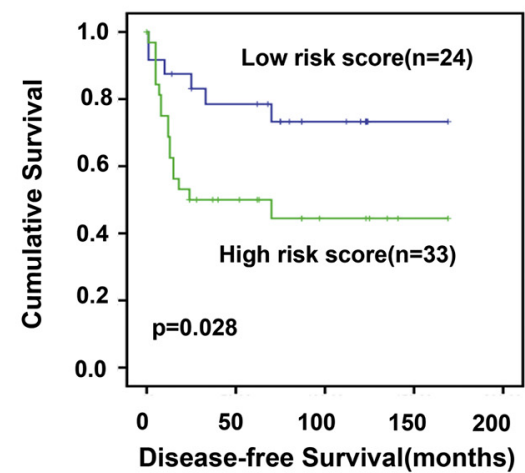

C TNM stage 3 with chemotherapy

$\begin{array}{llllll}\text { No. at risk } & & & & \\ \text { Low risk score } & 24 & 18 & 11 & 3 & 1 \\ \text { High risk score } & 32 & 13 & 6 & 2 & 1\end{array}$

d. TNM stage 2 with chemotherapy

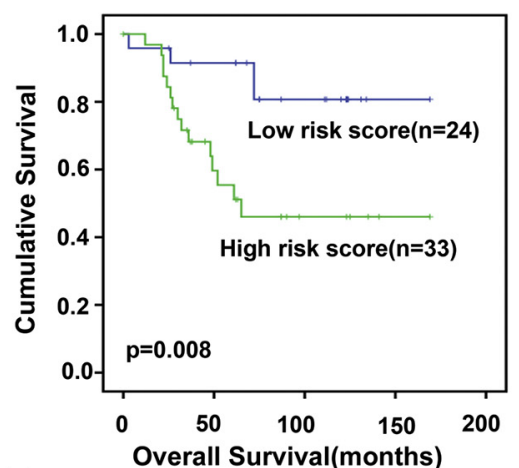

No. at risk

$\begin{array}{llllll}\text { Low risk score } & 24 & 21 & 13 & 2 & 1 \\ \text { High risk score } & 32 & 15 & 6 & 2 & 1\end{array}$
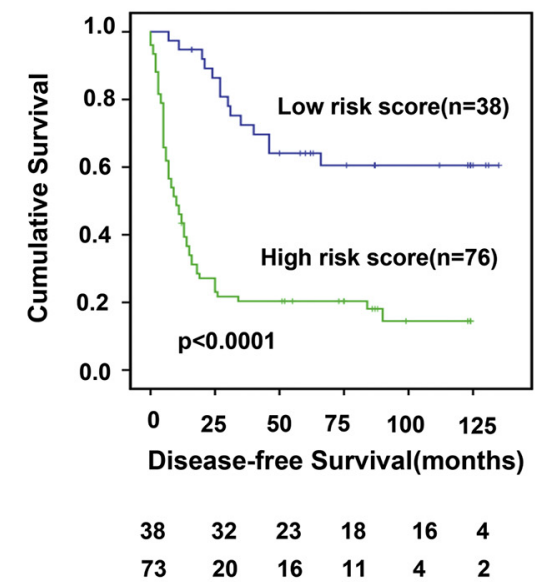

e TNM stage 3 with chemotherapy

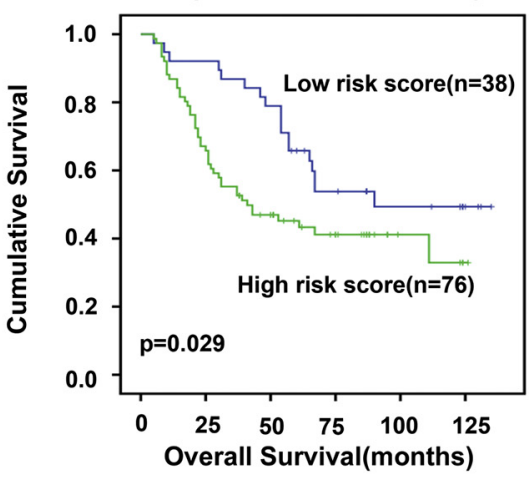

$\begin{array}{cccccc}38 & 36 & 31 & 20 & 12 & 4 \\ 76 & 51 & 32 & 18 & 6 & 3\end{array}$

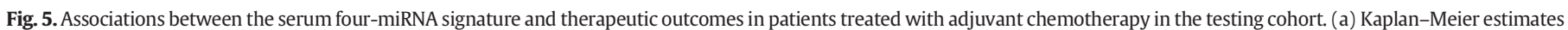

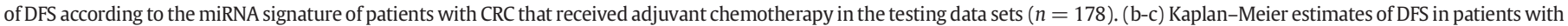
stage II or III CRC that received adjuvant chemotherapy. (d-e) Kaplan-Meier estimates of OS in patients with stage II or III CRC that received adjuvant chemotherapy.

The diversity of potential miRNA markers might be mainly due to the differences in the composition of cohort and selection methods of candidates between different studies. In some studies, the training cohort included healthy controls, colorectal adenomas and cancers with stage I-IV $[19,25]$. The candidate miRNAs were selected by comparing the miRNA levels among different groups and selecting the significantly dysregulated miRNAs. In attempt to predict the prognosis at the time of diagnosis, the patients enrolled in our study were stage I-III CRC patients. In the training data set, the Cox proportional hazards regression analyses was used to find miRNAs associated with patient survival.
Most of previous studies were based on preselected miRNA panels or several miRNAs selected depending function analysis [21,22,24]. Based on the profiling of 95 preselected miRNAs, miR-122 was verified to be associated with a 'bad' prognostic subtype in metastatic CRC and a shorter relapse-free survival and overall survival for CRC patients [20]. The work of Vychytilova-Faltejskova P et al. was based on genome-wide miRNA profiling, but the profiling was performed using pooled samples of colon cancer patients/healthy donors [25]. Our study used serum/plasma focused miRNA PCR Panel including 175 miRNAs. 
The platforms applied during discovery stage were different. The platforms applied for quantification of miRNA expression included RNA sequencing [25], reverse-transcription-quantitative PCR (RT-PCR) [20] and microarray hybridization [19]. Each method has its strengths and weaknesses. There is only $54.6 \%$ average validation rate for differentially expressed miRNAs between any two platform combinations. The hybridization-based platform shows lower sensitivity, even when input RNA is not limiting, whereas sequencing platform is sensitive when the amount of RNA is not limiting. Low-input-amount RNA used for sequencing platform may reduce the sensitivity. Serum is cell free liquid sample and only the circulating RNA is extracted, resulting in low total RNA concentrations. To characterize miRNA signatures in the serum of CRC, we chose the qPCR platforms which has superior sensitivity and accuracy [26].

The different studies utilized different methods for normalization. In some studies, synthetic $C$. elegans mir-39 was used as reference miRNA $[19,20,22,24]$. In our study, we used the selected endogenous control miR-103-3p and miR-93-5p, which was similar with - VychytilovaFaltejskova P' s study [25].

Several miRNAs were shown to be differentially expressed between the chemosensitive and chemoresistant patients [27-29]. These studies often enrolled both non-metastatic and metastatic CRC patients. The patients enrolled in our study were stage I-III. Our four- miRNA signature was associated with therapeutic outcome in stage I-III CRC patients who received adjuvant therapy.

Some researches indicated that the circulating miRNAs are not only biomarkers for CRC recurrence and metastasis but are also involved in regulating the biological functions of CRC by modulating the local tumor microenvironment or the immune system response [30,31].

These four miRNAs and their related targets are involved in regulating various properties of cancer, such as cancer-drug resistance by drug efflux, regulation of actin-based motility by Rho, actin cytoskeleton signaling, integrin signaling, and HMGB1 signaling pathways. The biological roles of the miR-652-3p and miR-501-3p have not been clarified in CRC as their roles in cancer development have been complicated. In pancreatic cancer, the miR-652 reportedly inhibited tumor growth and liver metastasis by targeting Zinc Finger E-Box Binding Homeobox 1 (ZEB1) [32], while in triple-negative breast cancer, higher circulating levels of the miR-652 were associated with an increased risk of recurrence and decreased OS [33]. High miR-501 expression is associated with recurrence in pancreatic head cancer [34]. Single nucleotide polymorphisms (rs112489955) located in miR-501 is associated with the risk of hepatocellular carcinoma in a Chinese Han population [35]. IPA predicted that TMEM204 was a target of the miR-652-3p and miR501-3p. Analysis of TCGA miRNA-seq and mRNA-seq data showed that the expression of TMEM204 was inversely correlated with miR-652$3 p$ and miR-501-3p and negatively associated with OS of CRC (data from Human Pathology Atlas data-www.proteinatlas.org/pathology). TMEM204 is a hypoxia-regulated transmembrane protein and plays a role in cell adhesion and cellular permeability at adherens junctions [36]. It also can interact with the VEGFR-2 and VEGFR-3 pathways and regulates lymphatic vessel development [37]. Synovial Sarcoma X Family Member 1 (SSX1) was predicted as a target of the miR-501-3p. As reported, SS18-SSX1 oncogenic fusion usurps Switch/Sucrose NonFermentable (SWI/SNF)-like BRG1 associated factor (BAF) complexes, resulting in activation of SRY-related HMG-box (SOX2), which drove the proliferation [38].

miR-328 was downregulated in CRC; low miR-328 expression tends to correlate with high Side population (SP) fraction. miR-328 overexpression reversed drug resistance and inhibited cell invasion of SP cells by targeting ATP Binding Cassette Subfamily G Member 2 (ABCG2) and Matrix Metallopeptidase 16 (MMP16) [39]. miR-342 was downregulated in CRC tissues and has been implicated in inhibiting tumor growth and lung metastasis by targeting DNA Methyltransferase 1 (DNMT1), Forkhead Box M1 (FOXM1), and Forkhead Box Q1 (FOXQ1)
[40]. SP140 was predicted as a target of miR-342-3p. The expression of SP140 was correlated with miR-342-3p and positively associated with the prognosis of CRC (data from Human Pathology Atlas data-www. proteinatlas.org/pathology). SP140 is a key regulator of macrophage transcriptional programs for cellular state and normally critical for intestinal homeostasis [41].

Although the mechanisms of miRNA secretion are unclear, circulating miRNAs seem to play a pivotal role as signaling molecules in cancer. Circulating miRNAs are not only biomarkers for CRC recurrence and metastasis, but are also involved in regulating the biological functions of CRC. These four miRNAs could inhibit CRC progress by affecting their targets network.

Altogether, our data indicate that the group of patients with high risk scores had a poor prognosis and did not benefit from current adjuvant chemotherapy. As a result, testing the expression levels of these serum biomarkers will help identify subgroups of patients who should be included in novel randomized clinical trials for alternative treatment strategies.

Our study has limitations. As the results are based on retrospective data, and as such prone to selection bias. This aspect is highlighted by our exclusion of patients in whom complete followup information was not available or had no blood sample from prior to treatments or the blood samples were hemolytic. Thus, future clinical trials should be prospectively conducted in multiple centers to evaluate the predictive accuracy of the serum four-miRNA signature. Although the serum miRNA signature appeared to be a better marker than CEA for CRC recurrence prediction based on ROC analysis, the ROC curve areas was 0.7427 . It will get better to further improve the specificity and sensitivity of prediction. In the future work, it is necessary to improve the predictive power of the signature through the expansion of samples, multi center validation, and adding other factors to stratify. The underlying mechanisms involved in the release of miRNAs into the blood stream of patients remain unclear. We will further investigate whether the deregulated miRNAs are from microvesicles, exosomes, circulating tumor cells, or apoptotic bodies.

\section{Funding}

This work was supported by the National Natural Science Foundation of China (81372593, 81201965, 81772565), Beijing Natural Science Foundation (7132052), and the National High Technology Research and Development Program of China (863 Program) (No.2012AA02A506, 2014AA020801).

\section{Conflict of interest}

The authors declare no conflict of interest.

\section{Authors' contributions}

DBJ designed and conducted experiments and wrote the manuscript. MQ performed RNA isolation from serum samples, qRT-PCR assay and data interpretation. YFY performed blood sample collection of training set and validation cohort, and clinical data analyses. HLC and QD performed blood sample collection of independent cohort and clinical data analyses. ML, JYJ, XXC, ZWL and JHX performed RNA isolation, qRT-PCR assay and data validation and analyses. JG designed and conducted experiments, analyzed data, acquired data, and wrote the manuscript. All authors read and approved the final manuscript.

\section{Acknowledgments}

The authors would like to thank Dr. Dana Meranus from Battelle Memory Institute for her professional statistical suggestions. 


\section{Appendix A. Supplementary data}

Supplementary data to this article can be found online at https://doi. org/10.1016/j.ebiom.2018.08.042.

\section{References}

[1] Vaiopoulos AG, Kostakis ID, Koutsilieris M, Papavassiliou AG. Colorectal cancer stem cells. Stem Cells 2012;30(3):363-71.

[2] Becerra AZ, Probst CP, Tejani MA, Aquina CT, Gonzalez MG, Hensley BJ, et al. Evaluating the prognostic role of elevated preoperative carcinoembryonic antigen levels in colon cancer patients: Results from the national cancer database. Ann Surg Oncol 2016;23(5):1554-61.

[3] Yousef M, Trinh $\mathrm{H}$, Allmer J. Intersection of MicroRNA and gene regulatory networks and their implication in cancer. Curr Pharm Biotechnol 2014;15(5):445-54.

[4] Si H, Sun X, Chen Y, Cao Y, Chen S, Wang H, et al. Circulating microRNA-92a and microRNA-21 as novel minimally invasive biomarkers for primary breast cancer. J Cancer Res Clin Oncol 2013;139(2):223-9.

[5] Clancy C, Joyce MR, Kerin MJ. The use of circulating microRNAs as diagnostic biomarkers in colorectal cancer. Cancer Biomark 2015;15(2):103-13.

[6] Wang J, Zhang KY, Liu SM, Sen S. Tumor-associated circulating microRNAs as biomarkers of cancer. Molecules 2014;19(2):1912-38.

[7] Ng EK, Chong WW, Jin H, Lam EK, Shin VY, Yu J, et al. Differential expression of microRNAs in plasma of patients with colorectal cancer: A potential marker for colorectal cancer screening. Gut 2009;58(10):1375-81.

[8] Huang Z, Huang D, Ni S, Peng Z, Sheng W, Du X. Plasma microRNAs are promising novel biomarkers for early detection of colorectal cancer. Int J Cancer 2010; 127(1):118-26.

[9] Fesler A, Jiang J, Zhai H, Ju J. Circulating microRNA testing for the early diagnosis and follow-up of colorectal cancer patients. Mol Diagn Ther 2014;18(3):303-8.

[10] Harboe M. A method for determination of hemoglobin in plasma by near-ultraviolet spectrophotometry. Scand J Clin Lab Invest 1959;11:66-70.

[11] Lippi G, Salvagno GL, Montagnana M, Brocco G, Guidi GC. Influence of hemolysis on routine clinical chemistry testing. Clin Chem Lab Med 2006;44(3):311-6.

[12] Bustin SA, Benes V, Garson JA, Hellemans J, Huggett J, Kubista M, et al. The MIQE guidelines: Minimum information for publication of quantitative real-time PCR experiments. Clin Chem 2009;55(4):611-22.

[13] Kramer A, Green J, Pollard Jr J, Tugendreich S. Causal analysis approaches in ingenuity pathway analysis. Bioinformatics 2014;30(4):523-30.

[14] Espinosa-Parrilla Y, Munoz X, Bonet C, Garcia N, Vencesla A, Yiannakouris N, et al. Genetic association of gastric cancer with miRNA clusters including the cancerrelated genes MIR29, MIR25, MIR93 and MIR106: Results from the EPIC-EURGAST study. Int J Cancer 2014;135(9):2065-76.

[15] Yu SL, Chen HY, Chang GC, Chen CY, Chen HW, Singh S, et al. MicroRNA signature predicts survival and relapse in lung cancer. Cancer Cell 2008:13(1):48-57.

[16] Punt CJ, Buyse M, Kohne CH, Hohenberger P, Labianca R, Schmoll HJ, et al. Endpoints in adjuvant treatment trials: A systematic review of the literature in colon cance and proposed definitions for future trials. J Natl Cancer Inst 2007;99(13):998-1003.

[17] Gill S, Loprinzi CL, Sargent DJ, Thome SD, Alberts SR, Haller DG, et al. Pooled analysis of fluorouracil-based adjuvant therapy for stage II and III colon cancer: Who benefits and by how much? J Clin Oncol 2004;22(10):1797-806

[18] Carey LA, Berry DA, Cirrincione CT, Barry WT, Pitcher BN, Harris LN, et al. Molecula Heterogeneity and Response to Neoadjuvant Human Epidermal Growth Factor Receptor 2 Targeting in CALGB 40601, a Randomized Phase III Trial of Paclitaxel Plus Trastuzumab With or Without Lapatinib. J Clin Oncol 2016;34(6):542-9.

[19] Imaoka H, Toiyama Y, Fujikawa H, Hiro J, Saigusa S, Tanaka K, et al. Circulating microRNA-1290 as a novel diagnostic and prognostic biomarker in human colorectal cancer. Ann Oncol 2016;27(10):1879-86.

[20] Maierthaler M, Benner A, Hoffmeister M, Surowy H, Jansen L, Knebel P, et al. Plasma miR-122 and miR-200 family are prognostic markers in colorectal cancer. Int J Cancer 2017; 140(1):176-87.
[21] Toiyama Y, Takahashi M, Hur K, Nagasaka T, Tanaka K, Inoue Y, et al. Serum miR-21 as a diagnostic and prognostic biomarker in colorectal cancer. J Natl Cancer Inst 2013;105(12):849-59.

[22] Hur K, Toiyama Y, Okugawa Y, Ide S, Imaoka H, Boland CR, et al. Circulating microRNA-203 predicts prognosis and metastasis in human colorectal cancer. Gut 2017;66(4):654-65.

[23] Yamada N, Nakagawa Y, Tsujimura N, Kumazaki M, Noguchi S, Mori T, et al. Role of Intracellular and Extracellular MicroRNA-92a in Colorectal Cancer. Transl Oncol 2013;6(4):482-92

[24] Toiyama Y, Hur K, Tanaka K, Inoue Y, Kusunoki M, Boland CR, et al. Serum miR-200c is a novel prognostic and metastasis-predictive biomarker in patients with colorectal cancer. Ann Surg 2014;259(4):735-43.

[25] Vychytilova-Faltejskova P, Radova L, Sachlova M, Kosarova Z, Slaba K, Fabian P, et al. Serum-based microRNA signatures in early diagnosis and prognosis prediction of colon cancer. Carcinogenesis 2016;37(10):941-50.

[26] Mestdagh P, Hartmann N, Baeriswyl L, Andreasen D, Bernard N, Chen C, et al. Evaluation of quantitative miRNA expression platforms in the microRNA quality control (miRQC) study. Nat Methods 2014;11(8):809-15.

[27] Kjersem JB, Ikdahl T, Lingjaerde OC, Guren T, Tveit KM, Kure EH. Plasma microRNAs predicting clinical outcome in metastatic colorectal cancer patients receiving firstline oxaliplatin-based treatment. Mol Oncol 2014;8(1):59-67.

[28] Chen O, Xia HW, Ge XJ, Zhang YC, Tang OL, Bi F. Serum miR-19a predicts resistance to FOLFOX chemotherapy in advanced colorectal cancer cases. Asian Pacific journal of cancer prevention. APJCP 2013;14(12):7421-6.

[29] Zhang J, Zhang K, Bi M, Jiao X, Zhang D, Dong Q. Circulating microRNA expressions in colorectal cancer as predictors of response to chemotherapy. Anticancer Drugs 2014; 25(3):346-52.

[30] Akao Y, Iio A, Itoh T, Noguchi S, Itoh Y, Ohtsuki Y, et al. Microvesicle-mediated RNA molecule delivery system using monocytes/macrophages. Mol Ther 2011;19(2): 395-9.

[31] Patel SA, Gooderham NJ. IL6 mediates immune and colorectal cancer cell cross-talk via miR-21 and miR-29b. Mol Cancer Res 2015;13(11):1502-8.

[32] Deng S, Li X, Niu Y, Zhu S, Jin Y, Deng S, et al. MiR-652 inhibits acidic microenvironment-induced epithelialmesenchymal transition of pancreatic cancer cells by targeting ZEB1. Oncotarget 2015;6(37).

[33] Kleivi Sahlberg K, Bottai G, Naume B, Burwinkel B, Calin GA, Borresen-Dale AL et al. A serum microRNA signature predicts tumor relapse and survival in triple-negative breast cancer patients. Clin Cancer Res 2015;21(5):1207-14

[34] Ling Q, Xu X, Ye P, Xie H, Gao F, Hu Q, et al. The prognostic relevance of primary tumor location in patients undergoing resection for pancreatic ductal adenocarcinoma. Oncotarget 2017;8(9):15159-67.

[35] Liu Y, Chai Y, Zhang J, Tang J. A function variant at miR-501 alters susceptibility to hepatocellular carcinoma in a chinese han population. Cell Physiol Biochem 2016; 38(6):2500-8

[36] Kearsey J, Petit S, De Oliveira C, Schweighoffer F. A novel four transmembrane spanning protein, CLP24. A hypoxically regulated cell junction protein. Eur J Biochem 2004·271(13):2584-92.

[37] Saharinen P, Helotera H, Miettinen J, Norrmen C, D'Amico G, Jeltsch M, et al. Claudinlike protein 24 interacts with the VEGFR-2 and VEGFR-3 pathways and regulates lymphatic vessel development. Genes Dev 2010;24(9):875-80.

[38] Kadoch C, Crabtree GR. Reversible disruption of mSWI/SNF (BAF) complexes by the SS18-SSX oncogenic fusion in synovial sarcoma. Cell 2013;153(1):71-85.

[39] Xu XT, Xu Q, Tong JL, Zhu MM, Nie F, Chen X, et al. MicroRNA expression profiling identifies miR-328 regulates cancer stem cell-like SP cells in colorectal cancer. $\mathrm{Br} J$ Cancer 2012;106(7):1320-30

[40] Wang H, Wu J, Meng X, Ying X, Zuo Y, Liu R, et al. MicroRNA-342 inhibits colorectal cancer cell proliferation and invasion by directly targeting DNA methyltransferase 1 . Carcinogenesis 2011;32(7):1033-42.

[41] Mehta S, Cronkite DA, Basavappa M, Saunders TL, Adiliaghdam F, Amatullah H, et al. Maintenance of macrophage transcriptional programs and intestinal homeostasis by epigenetic reader SP140. Sci Immunol 2017;2(9). 